\title{
Riemann-Hilbert approach and $N$-soliton solution for an eighth-order nonlinear Schrödinger equation in an optical fiber
}

Zhou-Zheng Kang ${ }^{1,2}$, Tie-Cheng $\mathrm{Xia}^{1 *}$ and Wen-Xiu Ma ${ }^{3,4}$

"Correspondence: xiatc@shu.edu.cn 'Department of Mathematics, Shanghai University, Shanghai, China

Full list of author information is available at the end of the article

\begin{abstract}
This paper aims to present an application of the Riemann-Hilbert approach to treat higher-order nonlinear differential equation that is an eighth-order nonlinear Schrödinger equation arising in an optical fiber. Starting from the spectral analysis of the Lax pair, a matrix Riemann-Hilbert problem is formulated strictly. Then, by solving the obtained Riemann-Hilbert problem under the reflectionless case, $\mathrm{N}$-soliton solution is generated for the eighth-order nonlinear Schrödinger equation. Finally, the localized structures and dynamic behaviors of one- and two-soliton solutions are illustrated by some figures.
\end{abstract}

MSC: $35 \mathrm{C08}$

Keywords: Eighth-order nonlinear Schrödinger equation; Riemann-Hilbert approach; Soliton solutions

\section{Introduction}

The infinite integrable nonlinear Schrödinger (NLS) equation hierarchy [1] reads as

$$
i p_{t}+A_{2} K_{2}[p(x, t)]-i A_{3} K_{3}[p(x, t)]+A_{4} K_{4}[p(x, t)]-i A_{5} K_{5}[p(x, t)]+\cdots=0
$$

which is used to investigate the higher-order dispersive effects and nonlinearity. Here $p(x, t)$ denotes a normalized complex amplitude of the optical pulse envelope. The coefficients $A_{l}$ are arbitrary real constants, and $K_{l}[p(x, t)]$ are the $l$ th-order operators in the NLS hierarchy

$$
\begin{aligned}
K_{2}[p(x, t)] & =p_{x x}+2 p|p|^{2}, \\
K_{3}[p(x, t)] & =p_{x x x}+6|p|^{2} p_{x} \\
K_{4}[p(x, t)] & =p_{x x x x}+6 p^{*} p_{x}^{2}+4 p\left|p_{x}\right|^{2}+8|p|^{2} p_{x x}+2 p^{2} p_{x x}^{*}+6|p|^{4} p \\
K_{5}[p(x, t)] & =p_{x x x x x}+10|p|^{2} p_{x x x}+30|p|^{4} p_{x}+10 p p_{x} p_{x x}^{*}+10 p p_{x}^{*} p_{x x} \\
& +20 p^{*} p_{x} p_{x x}+10 p_{x}^{2} p_{x}^{*}
\end{aligned}
$$

(c) The Author(s) 2019. This article is distributed under the terms of the Creative Commons Attribution 4.0 International License (http://creativecommons.org/licenses/by/4.0/), which permits unrestricted use, distribution, and reproduction in any medium, provided you give appropriate credit to the original author(s) and the source, provide a link to the Creative Commons license, and indicate if changes were made. 
Here the subscripts of $p(x, t)$ mean the partial derivatives with respect to the scaled spatial coordinate $x$ and time coordinate $t$ correspondingly. And the superscript $*$ represents complex conjugate.

As a matter of fact, Equation (1) covers many nonlinear differential equations of important significance, some of which are listed as follows:

(i) For the case of $A_{l}=0, l \geq 3$, Equation (1) is reduced to the fundamental nonlinear Schrödinger equation describing the propagation of the picosecond pulses in an optical fiber.

(ii) For the case of $A_{2}=\frac{1}{2}$ and $A_{l}=0, l \geq 4$, Equation (1) is reduced to the Hirota equation [2-5] describing the third-order dispersion and time-delay correction to the cubic nonlinearity in ocean waves.

(iii) For the case of $A_{2}=\frac{1}{2}$ and $A_{l}=0, l \geq 5$, Equation (1) becomes a fourth-order dispersive NLS equation $[6,7]$ describing the ultrashort optical-pulse propagation in a long-distance, high-speed optical fiber transmission system.

(iv) For the case of $A_{2}=\frac{1}{2}$ and $A_{l}=0, l \geq 6$, Equation (1) becomes a fifth-order NLS equation [8] describing the attosecond pulses in an optical fiber.

In recent years, researchers have devoted their attention to many higher-order NLS equations truncating from Equation (1). For instance, an eighth-order NLS equation was under study [9]. The interactions among multiple solitons were discussed, and oscillations in the interaction zones were observed systematically. As a result, it was found that the oscillations in the solitonic interaction zones possess different forms with different spectral parameters and so forth. In a follow-up study [10], the Lax pair and infinitely-many conservation laws were derived via symbolic computation, which verifies the integrability of equation.

All the time, seeking exact solutions of nonlinear models is of an especially important significance in the study of various nonlinear phenomena [11-15]. With this in mind, in this paper, we investigate in detail an eighth-order NLS equation [16]

$$
\begin{aligned}
i p_{t} & +p_{x x x x x x x x}+16|p|^{2} p_{x x x x x x}+2 p^{2} p_{x x x x x x}^{*}+56 p^{*} p_{x} p_{x x x x x}+40 p p_{x}^{*} p_{x x x x x} \\
& +12 p p_{x} p_{x x x x x}^{*}+98|p|^{4} p_{x x x x}+168\left|p_{x}\right|^{2} p_{x x x x}+112 p^{*} p_{x x} p_{x x x x}+72 p p_{x x}^{*} p_{x x x x} \\
& +28 p^{2}|p|^{2} p_{x x x x}^{*}+42 p_{x}^{2} p_{x x x x}^{*}+44 p p_{x x} p_{x x x x}^{*}+68 p p_{x x x} p_{x x x}^{*}+476|p|^{2} p^{*} p_{x} p_{x x x} \\
& +252 p_{x} p_{x x}^{*} p_{x x x}+308 p|p|^{2} p_{x}^{*} p_{x x x}+308 p_{x}^{*} p_{x x} p_{x x x}+70 p^{*} p_{x x x}^{2}+196 p_{x} p_{x x} p_{x x x}^{*} \\
& +168 p|p|^{2} p_{x} p_{x x x}^{*}+56 p^{3} p_{x}^{*} p_{x x x}^{*}+280|p|^{6} p_{x x}+1456|p|^{2}\left|p_{x}\right|^{2} p_{x x}+490\left(p^{*}\right)^{2} p_{x}^{2} p_{x x} \\
& +238 p^{2}\left(p_{x}^{*}\right)^{2} p_{x x}+588|p|^{2} p_{x}^{2} p_{x x}^{*}+336 p^{2}\left|p_{x}\right|^{2} p_{x x}^{*}+140|p|^{4} p^{2} p_{x x}^{*}+42 p^{3}\left(p_{x x}^{*}\right)^{2} \\
& +392|p|^{2} p\left|p_{x x}\right|^{2}+322|p|^{2} p^{*} p_{x x}^{2}+182 p_{x x}^{2} p_{x x}^{*}+560|p|^{4} p^{*} p_{x}^{2}+560|p|^{4} p\left|p_{x}\right|^{2} \\
& +420 p^{*} p_{x}^{2}\left|p_{x}\right|^{2}+140 p^{3}|p|^{2}\left(p_{x}^{*}\right)^{2}+378\left|p_{x}\right|^{4} p+70|p|^{8} p=0
\end{aligned}
$$

which works as a model for describing the propagation of ultrashort nonlinear pulses. The same scalar equation can be found from Equation (1) where $K_{l}(x, t)$ have the same meaning as $H_{l+1}\left(p,-p^{*}\right)$. Here $p(x, t)$ denotes a normalized complex amplitude of the optical pulse envelope.

The principal aim of this paper is to determine multi-soliton solutions for the eighthorder NLS equation (2) with the aid of the Riemann-Hilbert approach [17-29]. This paper 
is divided into five sections. In the second section, we recall the Lax pair associated with Equation (2) and convert it into a more convenient form. In the third section, we carry out the spectral analysis, from which a matrix Riemann-Hilbert problem is set up on the real axis. In the fourth section, the construction of multi-soliton solutions for Equation (2) is detailedly discussed in the framework of the Riemann-Hilbert problem without reflection. A brief conclusion is given in the final section.

\section{Lax pair}

Upon the Ablowitz-Kaup-Newell-Segur formalism, Equation (2) is associated with the following Lax pair [16]:

$$
\begin{aligned}
& \Psi_{x}=U \Psi, \quad U=i \varsigma \sigma+i Q, \\
& \Psi_{\tilde{t}}=V \Psi, \quad V=128 i \varsigma^{8} \sigma+128 i \varsigma^{7} Q+\sum_{k=1}^{7}(2 \varsigma)^{7-k} V_{k}^{0},
\end{aligned}
$$

where $\Psi=\left(\Psi_{1}, \Psi_{2}\right)^{\mathrm{T}}$ is a vector eigenfunction, $\Psi_{1}$ and $\Psi_{2}$ are the complex functions of $x$ and $t$, the symbol $\mathrm{T}$ signifies transpose of the vector, and $\varsigma$ is an isospectral parameter. Furthermore,

$$
\sigma=\left(\begin{array}{cc}
-1 & 0 \\
0 & 1
\end{array}\right), \quad Q=\left(\begin{array}{cc}
0 & p \\
-q & 0
\end{array}\right), \quad V_{k}^{0}=\left(\begin{array}{cc}
-i^{k} F_{k}(p, q) & i^{k-1} H_{k}(p, q) \\
i^{k-1} G_{k}(p, q) & i^{k} F_{k}(p, q)
\end{array}\right)
$$

and

$$
\begin{aligned}
& H_{1}(p, q)=-p_{x}, \quad G_{1}(p, q)=-q_{x}, \quad\left(F_{k}(p, q)\right)_{x}=-p G_{k}(p, q)-q H_{k}(p, q), \\
& H_{k+1}(p, q)=2 p F_{k}(p, q)+\left(H_{k}(p, q)\right)_{x}, \quad G_{k+1}(p, q)=-2 q F_{k}(p, q)-\left(G_{k}(p, q)\right)_{x} .
\end{aligned}
$$

Particularly,

$$
\begin{aligned}
& F_{1}(p, q)=p q, \quad H_{2}(p, q)=2 p^{2} q-p_{x x}, \\
& G_{2}(p, q)=-2 q^{2} p+q_{x x}, \quad F_{2}(p, q)=p_{x} q-p q_{x} \\
& H_{3}(p, q)=6 p q p_{x}-p_{x x x}, \quad G_{3}(p, q)=6 p q q_{x}-q_{x x x}, \\
& F_{3}(p, q)=p q_{x x}+q p_{x x}-p_{x} q_{x}-3 p^{2} q^{2} \\
& H_{4}(p, q)=-6 p^{3} q^{2}+6 q p_{x}^{2}+4 p p_{x} q_{x}+8 p q p_{x x}+2 p^{2} q_{x x}-p_{x x x x}, \\
& G_{4}(p, q)=6 p^{2} q^{3}-6 p q_{x}^{2}-4 q p_{x} q_{x}-8 p q q_{x x}-2 q^{2} p_{x x}+q_{x x x x}, \\
& F_{4}(p, q)=-6 p q^{2} p_{x}+6 p^{2} q q_{x}-p_{x x} q_{x}+p_{x} q_{x x}+q p_{x x x}-p q_{x x x}, \\
& H_{5}(p, q)=-30 p^{2} q^{2} p_{x}+10 p_{x}^{2} q_{x}+20 q p_{x} p_{x x}+10 p q_{x} p_{x x}+10 p p_{x} q_{x x}+10 p q p_{x x x}-p_{x x x x x}, \\
& G_{5}(p, q)=-30 p^{2} q^{2} q_{x}+10 q_{x}^{2} p_{x}+10 q q_{x} p_{x x}+10 q p_{x} q_{x x}+10 p q q_{x x x}+20 p q_{x} q_{x x}-q_{x x x x x}, \\
& F_{5}(p, q)=10 p^{3} q^{3}-5 q^{2} p_{x}^{2}-5 p^{2} q_{x}^{2}-10 p q^{2} p_{x x}-10 p^{2} q q_{x x}+p_{x x} q_{x x}-q_{x} p_{x x x}-p_{x} q_{x x x} \\
& \quad+q p_{x x x x}+p q_{x x x x},
\end{aligned}
$$




$$
\begin{aligned}
& H_{6}(p, q)=20 p^{4} q^{3}-70 p q^{2} p_{x}^{2}-60 p^{2} q p_{x} q_{x}-10 p^{3} q_{x}^{2}-50 p^{2} q^{2} p_{x x}+50 p_{x} q_{x} p_{x x}+20 q p_{x x}^{2} \\
& -20 p^{3} q q_{x x}+20 p_{x}^{2} q_{x x}+22 p p_{x x} q_{x x}+30 q p_{x} p_{x x x}+18 p q_{x} p_{x x x}+8 p p_{x} q_{x x x} \\
& +12 p q p_{x x x x}+2 p^{2} q_{x x x x}-p_{x x x x x x} \\
& G_{6}(p, q)=-20 q^{4} p^{3}+10 q^{3} p_{x}^{2}+60 q^{2} p p_{x} q_{x}+70 q p^{2} q_{x}^{2}+20 q^{3} p p_{x x}-20 q_{x}^{2} p_{x x} \\
& +50 p^{2} q^{2} q_{x x}-50 p_{x} q_{x} q_{x x}-22 q p_{x x} q_{x x}-20 p q_{x x}^{2}-8 q q_{x} p_{x x x}-18 q p_{x} q_{x x x} \\
& -30 p q_{x} q_{x x x}-2 q^{2} p_{x x x x}-12 p q q_{x x x x}+q_{x x x x x x} \text {, } \\
& F_{6}(p, q)=30 p^{2} q^{3} p_{x}-30 p^{3} q^{2} q_{x}-10 q p_{x}^{2} q_{x}+10 p p_{x} q_{x}^{2}-20 q^{2} p_{x} p_{x x}+10 p q q_{x} p_{x x} \\
& -10 p q p_{x} q_{x x}+20 p^{2} q_{x} q_{x x}-10 p q^{2} p_{x x x}+q_{x x} p_{x x x}+10 p^{2} q q_{x x x}-p_{x x} q_{x x x} \\
& -q_{x} p_{x x x x}+p_{x} q_{x x x x}+q p_{x x x x x}-p q_{x x x x x} \\
& H_{7}(p, q)=140 p^{3} q^{3} p_{x}-70 q^{2} p_{x}^{3}-280 p q p_{x}^{2} q_{x}-70 p^{2} q_{x}^{2} p_{x}-280 p q^{2} p_{x} p_{x x}-140 p^{2} q q_{x} p_{x x} \\
& +70 q_{x} p_{x x}^{2}-140 p^{2} q p_{x} q_{x x}+112 p_{x} p_{x x} q_{x x}-70 p^{2} q^{2} p_{x x x}+98 p_{x} q_{x} p_{x x x} \\
& +70 q p_{x x} p_{x x x}+42 p q_{x x} p_{x x x}+28 p_{x}^{2} q_{x x x}+28 p p_{x x} q_{x x x}+42 q p_{x} p_{x x x x} \\
& +28 p q_{x} p_{x x x x}+14 p p_{x} q_{x x x x}+14 p q p_{x x x x x}-p_{x x x x x x x} \\
& G_{7}(p, q)=140 p^{3} q^{3} q_{x}-70 q^{2} p_{x}^{2} q_{x}-280 p q p_{x} q_{x}^{2}-70 p^{2} q_{x}^{3}-140 p q^{2} q_{x} p_{x x}-140 p q^{2} p_{x} q_{x x} \\
& -280 p^{2} q q_{x} q_{x x}+112 q_{x} p_{x x} q_{x x}+70 p_{x} q_{x x}^{2}+28 q_{x}^{2} p_{x x x}+28 q q_{x x} p_{x x x} \\
& -70 p^{2} q^{2} q_{x x x}+98 p_{x} q_{x} q_{x x x}+42 q p_{x x} q_{x x x}+70 p q_{x x} q_{x x x}-14 q q_{x} p_{x x x x} \\
& +28 q p_{x} q_{x x x x}+42 p q_{x} q_{x x x x}+14 p q q_{x x x x x}-q_{x x x x x x x} \\
& F_{7}(p, q)=-35 p^{4} q^{4}+70 p q^{3} p_{x}^{2}+70 p^{2} q^{2} p_{x} q_{x}+70 p^{3} q q_{x}^{2}+21 p_{x}^{2} q_{x}^{2}+70 p^{2} q^{3} p_{x x} \\
& -28 q p_{x} q_{x} p_{x x}-14 p q_{x}^{2} p_{x x}-21 q^{2} p_{x x}^{2}+70 p^{3} q^{2} q_{x x}-14 q p_{x}^{2} q_{x x}-28 p p_{x} q_{x} q_{x x} \\
& -56 p q p_{x x} q_{x x}-21 p^{2} q_{x x}^{2}-28 q^{2} p_{x} p_{x x x}-14 p q q_{x} p_{x x x}-14 p q p_{x} q_{x x x} \\
& -28 p^{2} q_{x} q_{x x x}-p_{x x x} q_{x x x}-14 p q^{2} p_{x x x x}+q_{x x} p_{x x x x}-14 p^{2} q q_{x x x x} \\
& +p_{x x} q_{x x x x}-q_{x} p_{x x x x x}-p_{x} q_{x x x x x}+q p_{x x x x x x}+p q_{x x x x x x} \text {. }
\end{aligned}
$$

Then the reductions $q=-p^{*}$ and $\tilde{t}=-t$ exactly result in Equation (2) based on the zerocurvature equations.

Let us now rewrite the Lax pair (3a)-(3b) in a more convenient form:

$$
\begin{aligned}
& \Psi_{x}=i(\varsigma \sigma+Q) \Psi, \\
& \Psi_{t}=-\left(128 i \varsigma^{8} \sigma+Q_{1}\right) \Psi,
\end{aligned}
$$

where

$$
Q=\left(\begin{array}{cc}
0 & p \\
p^{*} & 0
\end{array}\right), \quad Q_{1}=128 i \varsigma^{7} Q+\sum_{k=1}^{7}(2 \varsigma)^{7-k} V_{k}^{0}
$$




\section{Riemann-Hilbert problem}

In this section, we focus on putting forward a matrix Riemann-Hilbert problem for Equation (2). Now we assume that the potential function $p(x, t)$ in the Lax pair (4a) $-(4 \mathrm{~b})$ decays to zero sufficiently fast as $x \rightarrow \pm \infty$. It can be known from (4a)-(4b) that when $x \rightarrow \pm \infty$,

$$
\Psi \propto \mu \mathrm{e}^{i \varsigma \sigma x-128 i \varsigma^{8} \sigma t}
$$

which motivates us to introduce the variable transformation

$$
\Psi=\mu \mathrm{e}^{i \zeta \sigma x-128 i \varsigma^{8} \sigma t}
$$

Under this transformation, the Lax pair (4a)-(4b) can be changed into the form

$$
\begin{aligned}
& \mu_{x}=i \varsigma[\sigma, \mu]+U_{1} \mu, \\
& \mu_{t}=-128 i \varsigma^{8}[\sigma, \mu]-Q_{1} \mu,
\end{aligned}
$$

where $[\cdot, \cdot]$ is the matrix commutator and $U_{1}=i Q$. From (5a) $-(5 \mathrm{~b})$, we find that $\operatorname{tr}\left(U_{1}\right)=$ $\operatorname{tr}\left(Q_{1}\right)=0$.

In the direct scattering process, we will concentrate on the spectral problem (5a), and the $t$-dependence will be suppressed. We first introduce two matrix Jost solutions $\mu_{ \pm}$of (5a) expressed as a collection of columns

$$
\mu_{-}=\left(\left[\mu_{-}\right]_{1},\left[\mu_{-}\right]_{2}\right), \quad \mu_{+}=\left(\left[\mu_{+}\right]_{1},\left[\mu_{+}\right]_{2}\right),
$$

meeting the asymptotic conditions at large distances

$$
\begin{aligned}
& \mu_{-} \rightarrow \mathbb{I}, \quad x \rightarrow-\infty, \\
& \mu_{+} \rightarrow \mathbb{I}, \quad x \rightarrow+\infty .
\end{aligned}
$$

Here the subscripts of $\mu$ indicated refer to which end of the $x$-axis the boundary conditions are required for, and $\mathbb{I}$ stands for the identity matrix of size 2 . Actually, the solutions $\mu_{ \pm}$ are uniquely determined by the integral equations of Volterra type

$$
\begin{aligned}
& \mu_{-}=\mathbb{I}+\int_{-\infty}^{x} \mathrm{e}^{i_{\zeta} \sigma(x-y)} U_{1}(y) \mu_{-}(y, \varsigma) \mathrm{e}^{i \zeta \sigma(y-x)} \mathrm{d} y, \\
& \mu_{+}=\mathbb{I}-\int_{x}^{+\infty} \mathrm{e}^{i \zeta \sigma(x-y)} U_{1}(y) \mu_{+}(y, \varsigma) \mathrm{e}^{i \zeta \sigma(y-x)} \mathrm{d} y .
\end{aligned}
$$

After direct analysis on Equations (7a)-(7b), we can see that $\left[\mu_{-}\right]_{1},\left[\mu_{+}\right]_{2}$ are analytic for $\varsigma \in \mathbb{C}^{+}$and continuous for $\varsigma \in \mathbb{C}^{+} \cup \mathbb{R}$, while $\left[\mu_{+}\right]_{1},\left[\mu_{-}\right]_{2}$ are analytic for $\varsigma \in \mathbb{C}^{-}$and continuous for $\varsigma \in \mathbb{C}^{-} \cup \mathbb{R}$, where $\mathbb{C}^{-}$and $\mathbb{C}^{+}$are respectively the lower and upper half ऽ-planes:

$$
\mathbb{C}^{-}=\{\varsigma \in \mathbb{C} \mid \operatorname{Im}(\varsigma)<0\}, \quad \mathbb{C}^{+}=\{\varsigma \in \mathbb{C} \mid \operatorname{Im}(\varsigma)>0\} .
$$

Next we set out to study the properties of $\mu_{ \pm}$. In fact, it can be shown from Abel's identity and $\operatorname{tr}\left(U_{1}\right)=0$ that the determinants of $\mu_{ \pm}$are independent of the variable $x$. Evaluating 
$\operatorname{det} \mu_{-}$at $x=-\infty$ and $\operatorname{det} \mu_{+}$at $x=+\infty$, we get $\operatorname{det} \mu_{ \pm}=1$ for $\varsigma \in \mathbb{R}$. In addition, $\mu_{-} E$ and $\mu_{+} E$ are both fundamental solutions of (3a), where $E=\mathrm{e}^{i \zeta \sigma x}$, they are linearly dependent

$$
\mu_{-} E=\mu_{+} E S(\varsigma), \quad \varsigma \in \mathbb{R} .
$$

Here $S(\varsigma)=\left(s_{k j}\right)_{2 \times 2}$ is called the scattering matrix and $\operatorname{det} S(\varsigma)=1$. Furthermore, we find from the properties of $\mu_{ \pm}$that $s_{11}$ allows analytic extension to $\mathbb{C}^{+}$and $s_{22}$ analytically extends to $\mathbb{C}^{-}$.

A matrix Riemann-Hilbert problem is closely connected with two matrix functions: one is analytic in $\mathbb{C}^{+}$and the other is analytic in $\mathbb{C}^{-}$. In consideration of the analytic properties of $\mu_{ \pm}$, we set

$$
P_{1}(x, \varsigma)=\left(\left[\mu_{-}\right]_{1},\left[\mu_{+}\right]_{2}\right)(x, \varsigma),
$$

defining in $\mathbb{C}^{+}$, be an analytic function of $\varsigma$. And then, $P_{1}$ can be expanded into the asymptotic series at large- 5

$$
P_{1}=P_{1}^{(0)}+\frac{P_{1}^{(1)}}{\varsigma}+\frac{P_{1}^{(2)}}{\varsigma^{2}}+O\left(\frac{1}{\varsigma^{3}}\right), \quad \varsigma \rightarrow \infty
$$

Inserting expansion (10) into the spectral problem (5a) and equating terms with the same powers of 5 , we obtain

$$
i\left[\sigma, P_{1}^{(1)}\right]+U_{1} P_{1}^{(0)}=P_{1 x}^{(0)}, \quad i\left[\sigma, P_{1}^{(0)}\right]=0,
$$

which yields $P_{1}^{(0)}=\mathbb{I}$, namely $P_{1} \rightarrow \mathbb{I}$ as $\varsigma \in \mathbb{C}^{+} \rightarrow \infty$.

For establishing a matrix Riemann-Hilbert problem, the analytic counterpart of $P_{1}$ in $\mathbb{C}^{-}$is still needed to be given. Note that the adjoint scattering equation of (5a) reads as

$$
H_{x}=i \varsigma[\sigma, H]-H U_{1}
$$

and the inverse matrices of $\mu_{ \pm}$meet this adjoint equation. Then we express the inverse matrices of $\mu_{ \pm}$as a collection of rows

$$
\mu_{ \pm}^{-1}=\left(\begin{array}{l}
{\left[\mu_{ \pm}^{-1}\right]^{1}} \\
{\left[\mu_{ \pm}^{-1}\right]^{2}}
\end{array}\right)
$$

which obey the boundary conditions $\mu_{ \pm}^{-1} \rightarrow \mathbb{I}$ as $x \rightarrow \pm \infty$. It is easy to know from (8) that

$$
E^{-1} \mu_{-}^{-1}=R(\varsigma) E^{-1} \mu_{+}^{-1},
$$

where $R(\varsigma)=\left(r_{k j}\right)_{2 \times 2}=S^{-1}(\varsigma)$. Thus, the matrix function $P_{2}$ which is analytic for $\varsigma \in \mathbb{C}^{-}$ is constructed as

$$
P_{2}(x, \varsigma)=\left(\begin{array}{l}
{\left[\mu_{-}^{-1}\right]^{1}} \\
{\left[\mu_{+}^{-1}\right]^{2}}
\end{array}\right)(x, \varsigma) .
$$


Analogous to $P_{1}$, the very large- $\varsigma$ asymptotic behavior of $P_{2}$ turns out to be $P_{2} \rightarrow \mathbb{I}$ as $\varsigma \in \mathbb{C}^{-} \rightarrow \infty$.

Carrying (6) into Equation (8) gives rise to

$$
\left(\left[\mu_{-}\right]_{1},\left[\mu_{-}\right]_{2}\right)=\left(\left[\mu_{+}\right]_{1},\left[\mu_{+}\right]_{2}\right)\left(\begin{array}{cc}
s_{11} & s_{12} \mathrm{e}^{-2 i \zeta x} \\
s_{21} \mathrm{e}^{2 i \zeta x} & s_{22}
\end{array}\right),
$$

from which we have

$$
\left[\mu_{-}\right]_{1}=s_{11}\left[\mu_{+}\right]_{1}+s_{21} \mathrm{e}^{2 i \zeta x}\left[\mu_{+}\right]_{2} .
$$

Hence, $P_{1}$ is of the form

$$
P_{1}=\left(\left[\mu_{-}\right]_{1},\left[\mu_{+}\right]_{2}\right)=\left(\left[\mu_{+}\right]_{1},\left[\mu_{+}\right]_{2}\right)\left(\begin{array}{cc}
s_{11} & 0 \\
s_{21} \mathrm{e}^{2 i \zeta x} & 1
\end{array}\right) .
$$

On the other hand, via substituting (12) into Equation (13), we get

$$
\left(\begin{array}{l}
{\left[\mu_{-}^{-1}\right]^{1}} \\
{\left[\mu_{-}^{-1}\right]^{2}}
\end{array}\right)=\left(\begin{array}{cc}
r_{11} & r_{12} \mathrm{e}^{-2 i \varsigma x} \\
r_{21} \mathrm{e}^{2 i \varsigma x} & r_{22}
\end{array}\right)\left(\begin{array}{l}
{\left[\mu_{+}^{-1}\right]^{1}} \\
{\left[\mu_{+}^{-1}\right]^{2}}
\end{array}\right),
$$

from which we can express $\left[\mu_{-}^{-1}\right]^{1}$ as

$$
\left[\mu_{-}^{-1}\right]^{1}=r_{11}\left[\mu_{+}^{-1}\right]^{1}+r_{12} \mathrm{e}^{-2 i_{5 x}}\left[\mu_{+}^{-1}\right]^{2} .
$$

As a consequence, $P_{2}$ is written as

$$
P_{2}=\left(\begin{array}{l}
{\left[\mu_{-}^{-1}\right]^{1}} \\
{\left[\mu_{+}^{-1}\right]^{2}}
\end{array}\right)=\left(\begin{array}{cc}
r_{11} & r_{12} \mathrm{e}^{-2 i \zeta x} \\
0 & 1
\end{array}\right)\left(\begin{array}{l}
{\left[\mu_{+}^{-1}\right]^{1}} \\
{\left[\mu_{+}^{-1}\right]^{2}}
\end{array}\right) .
$$

With two matrix functions $P_{1}$ and $P_{2}$ which are analytic in $\mathbb{C}^{+}$and $\mathbb{C}^{-}$respectively in hand, we are in a position to deduce a matrix Riemann-Hilbert problem for Equation (2). After denoting that the limit of $P_{1}$ is $P^{+}$as $\varsigma \in \mathbb{C}^{+} \rightarrow \mathbb{R}$ and the limit of $P_{2}$ is $P^{-}$as $\varsigma \in \mathbb{C}^{-} \rightarrow \mathbb{R}$, a matrix Riemann-Hilbert problem can be given as follows:

$$
P^{-}(x, \varsigma) P^{+}(x, \varsigma)=\left(\begin{array}{cc}
1 & r_{12} \mathrm{e}^{-2 i \varsigma x} \\
s_{21} \mathrm{e}^{2 i \varsigma x} & 1
\end{array}\right)
$$

with its canonical normalization conditions as

$$
\begin{aligned}
& P_{1}(x, \varsigma) \rightarrow \mathbb{I}, \quad \varsigma \in \mathbb{C}^{+} \rightarrow \infty, \\
& P_{2}(x, \varsigma) \rightarrow \mathbb{I}, \quad \varsigma \in \mathbb{C}^{-} \rightarrow \infty,
\end{aligned}
$$

and $r_{11} s_{11}+r_{12} s_{21}=1$. 


\section{$4 \mathrm{~N}$-Soliton solution}

Having described a matrix Riemann-Hilbert problem for Equation (2), we now turn to seeking its multi-soliton solutions. To achieve the goal, we first need to solve the RiemannHilbert problem (15) under the assumption of irregularity, which signifies that both $\operatorname{det} P_{1}$ and $\operatorname{det} P_{2}$ possess some zeros in the analytic domains of their own. From the definitions of $P_{1}$ and $P_{2}$ as well as Equation (8), we have

$$
\begin{array}{ll}
\operatorname{det} P_{1}(\varsigma)=s_{11}(\varsigma), & \varsigma \in \mathbb{C}^{+}, \\
\operatorname{det} P_{2}(\varsigma)=r_{11}(\varsigma), & \varsigma \in \mathbb{C}^{-},
\end{array}
$$

which means that $\operatorname{det} P_{1}$ and $\operatorname{det} P_{2}$ have the same zeros as $s_{11}$ and $r_{11}$ respectively, and $r_{11}=\left(S^{-1}\right)_{11}=s_{22}$.

With the above analysis, it is now necessary to reveal the characteristic feature of zeros. Manifestly, the potential matrix $Q$ possesses the symmetry relation $Q^{\dagger}=Q$, upon which we deduce

$$
\mu_{ \pm}^{\dagger}\left(\varsigma^{*}\right)=\mu_{ \pm}^{-1}(\varsigma)
$$

Here the superscript $\dagger$ stands for the Hermitian of a matrix. For facilitating discussion, we introduce two special matrices $J_{1}=\operatorname{diag}(1,0)$ and $J_{2}=\operatorname{diag}(0,1)$, and express (9) and (14) in terms of

$$
\begin{aligned}
& P_{1}=\mu_{-} J_{1}+\mu_{+} J_{2}, \\
& P_{2}=J_{1} \mu_{-}^{-1}+J_{2} \mu_{+}^{-1} .
\end{aligned}
$$

A direct computation of the Hermitian of expression (17a), using relation (16), generates that

$$
P_{1}^{\dagger}\left(\varsigma^{*}\right)=P_{2}(\varsigma), \quad \varsigma \in \mathbb{C}^{-}
$$

and $S^{\dagger}\left(\varsigma^{*}\right)=S^{-1}(\varsigma)$, which leads to

$$
s_{11}^{*}\left(\varsigma^{*}\right)=r_{11}(\varsigma), \quad \varsigma \in \mathbb{C}^{-} .
$$

This equality implies that each zero $\pm \zeta_{k}$ of $s_{11}$ results in each zero $\pm \varsigma_{k}^{*}$ of $r_{11}$ correspondingly. Therefore, our assumption is that $\operatorname{det} P_{1}$ has simple zeros $\left\{\varsigma_{j} \in \mathbb{C}^{+}, 1 \leq j \leq N\right\}$ and $\operatorname{det} P_{2}$ has simple zeros $\left\{\hat{\zeta}_{j} \in \mathbb{C}^{-}, 1 \leq j \leq N\right\}$, where $\hat{\zeta}_{j}=\zeta_{j}^{*}$. The full set of the discrete scattering data is composed of these zeros and the nonzero column vectors $v_{j}$ and row vectors $\hat{v}_{j}$, which satisfy the following equations:

$$
\begin{aligned}
& P_{1}\left(\varsigma_{j}\right) v_{j}=0, \\
& \hat{v}_{j} P_{2}\left(\hat{\zeta}_{j}\right)=0 .
\end{aligned}
$$

Taking the Hermitian of Equation (20a) and using (18) as well as comparing with Equation (20b), we find that the eigenvectors fulfill the relation

$$
\hat{v}_{j}=v_{j}^{\dagger}, \quad 1 \leq j \leq N .
$$


Differentiating Equation (20a) in $x$ and $t$ and taking advantage of Lax pair (5a)-(5b), we arrive at

$$
\begin{aligned}
& P_{1}\left(\varsigma_{j}\right)\left(\frac{\partial v_{j}}{\partial x}-i \varsigma_{j} \sigma v_{j}\right)=0, \\
& P_{1}\left(\varsigma_{j}\right)\left(\frac{\partial v_{j}}{\partial t}+128 i \varsigma_{j}^{8} \sigma v_{j}\right)=0,
\end{aligned}
$$

which yields

$$
v_{j}=\mathrm{e}^{\left(i \zeta_{j} x-128 i \zeta_{j}^{8} t\right) \sigma} v_{j, 0}, \quad 1 \leq j \leq N .
$$

Here $v_{j, 0}, 1 \leq j \leq N$, are complex constant vectors. Making use of relation (21), we have

$$
\hat{v}_{j}=v_{j, 0}^{\dagger} \mathrm{e}^{\left(-i \zeta_{j}^{*} x+128 i 5_{j}^{* 8} t\right) \sigma}, \quad 1 \leq j \leq N
$$

However, in order to derive soliton solutions of Equation (2), we investigate the Riemann-Hilbert problem (15) corresponding to the reflectionless case, i.e., $s_{21}=0$. We introduce an $N \times N$ matrix $M$ defined as

$$
M=\left(M_{k j}\right)_{N \times N}=\left(\frac{\hat{v}_{k} v_{j}}{\zeta_{j}-\hat{\zeta}_{k}}\right)_{N \times N}, \quad 1 \leq k, j \leq N .
$$

Thus the solutions [30] to problem (15) can be determined by

$$
\begin{aligned}
& P_{1}(\varsigma)=\mathbb{I}-\sum_{k=1}^{N} \sum_{j=1}^{N} \frac{v_{k} \hat{v}_{j}\left(M^{-1}\right)_{k j}}{\varsigma-\hat{\zeta}_{j}}, \\
& P_{2}(\varsigma)=\mathbb{I}+\sum_{k=1}^{N} \sum_{j=1}^{N} \frac{v_{k} \hat{v}_{j}\left(M^{-1}\right)_{k j}}{\varsigma-\varsigma_{k}},
\end{aligned}
$$

where $\left(M^{-1}\right)_{k j}$ denotes the $(k, j)$-entry of $M^{-1}$. From expression (22a), it can be seen that

$$
P_{1}^{(1)}=-\sum_{k=1}^{N} \sum_{j=1}^{N} v_{k} \hat{v}_{j}\left(M^{-1}\right)_{k j} .
$$

In what follows, we shall retrieve the potential function $p(x, t)$ based on the scattering data. Expanding $P_{1}(\varsigma)$ at large- $\varsigma$ as

$$
P_{1}(\varsigma)=\mathbb{I}+\frac{P_{1}^{(1)}}{\varsigma}+\frac{P_{1}^{(2)}}{\varsigma^{2}}+O\left(\frac{1}{\varsigma^{3}}\right), \quad \varsigma \rightarrow \infty
$$

and carrying this expansion into (5a) gives rise to

$$
Q=-\left[\sigma, P_{1}^{(1)}\right] .
$$

Consequently, the potential function is reconstructed as

$$
p(x, t)=2\left(P_{1}^{(1)}\right)_{12}
$$

with $\left(P_{1}^{(1)}\right)_{12}$ being the $(1,2)$-entry of $P_{1}^{(1)}$. 
To conclude, setting the nonzero vectors $v_{k, 0}=\left(\alpha_{k}, \beta_{k}\right)^{\mathrm{T}}$ and $\theta_{k}=i \zeta_{k} x-128 i \zeta_{k}^{8} t$, the general $N$-soliton solution for the eighth-order NLS equation (2) is written as

$$
p(x, t)=-2 \sum_{k=1}^{N} \sum_{j=1}^{N} \alpha_{k} \beta_{j}^{*} \mathrm{e}^{\theta_{j}^{*}-\theta_{k}}\left(M^{-1}\right)_{k j},
$$

where

$$
M_{k j}=\frac{\alpha_{k}^{*} \alpha_{j} \mathrm{e}^{-\theta_{k}^{*}-\theta_{j}}+\beta_{k}^{*} \beta_{j} \mathrm{e}^{\theta_{k}^{*}+\theta_{j}}}{\varsigma_{j}-\varsigma_{k}^{*}}, \quad 1 \leq k, j \leq N
$$

The bright one- and two-soliton solutions will be our main concern in the rest of this section. For the simplest case of $N=1$, the bright one-soliton solution can be readily derived as

$$
p(x, t)=-2 \alpha_{1} \beta_{1}^{*} \mathrm{e}^{\theta_{1}^{*}-\theta_{1}} \frac{\varsigma_{1}-\varsigma_{1}^{*}}{\left|\alpha_{1}\right|^{2} \mathrm{e}^{-\theta_{1}^{*}-\theta_{1}}+\left|\beta_{1}\right|^{2} \mathrm{e}^{\theta_{1}^{*}+\theta_{1}}},
$$

where $\theta_{1}=i \varsigma_{1} x-128 i \varsigma_{1}^{8} t$. Furthermore, via fixing $\alpha_{1}=1$ and setting $\varsigma_{1}=\tilde{a}_{1}+i \tilde{b}_{1}$ as well as $\left|\beta_{1}\right|^{2}=\mathrm{e}^{2 \xi_{1}}$, the solution (24) is then turned into the following form:

$$
p(x, t)=-2 i \tilde{b}_{1} \beta_{1}^{*} \mathrm{e}^{\theta_{1}^{*}-\theta_{1}} \mathrm{e}^{-\xi_{1}} \operatorname{sech}\left(\theta_{1}^{*}+\theta_{1}+\xi_{1}\right),
$$

where

$$
\begin{aligned}
& \theta_{1}^{*}+\theta_{1}=-2 \tilde{b}_{1} x+2048 \tilde{a}_{1}^{7} \tilde{b}_{1} t-14336 \tilde{a}_{1}^{5} \tilde{b}_{1}^{3} t+14336 \tilde{a}_{1}^{3} \tilde{b}_{1}^{5} t-2048 \tilde{a}_{1} \tilde{b}_{1}^{7} t \\
& \theta_{1}^{*}-\theta_{1}=-2 i \tilde{a}_{1} x-7168 i \tilde{a}_{1}^{2} \tilde{b}_{1}^{6} t-7168 i \tilde{a}_{1}^{6} \tilde{b}_{1}^{2} t+17920 i \tilde{a}_{1}^{4} \tilde{b}_{1}^{4} t+256 i \tilde{b}_{1}^{8} t+256 i \tilde{a}_{1}^{8} t .
\end{aligned}
$$

Hence we can further write the bright one-soliton solution (25) as

$$
\begin{aligned}
p(x, t)= & -2 i \tilde{b}_{1} \beta_{1}^{*} \mathrm{e}^{\theta_{1}^{*}-\theta_{1}} \mathrm{e}^{-\xi_{1}} \\
& \times \operatorname{sech}\left\{-2 \tilde{b}_{1}\left[x-\left(1024 \tilde{a}_{1}^{7}-7168 \tilde{a}_{1}^{5} \tilde{b}_{1}^{2}+7168 \tilde{a}_{1}^{3} \tilde{b}_{1}^{4}-1024 \tilde{a}_{1} \tilde{b}_{1}^{6}\right) t\right]+\xi_{1}\right\},
\end{aligned}
$$

from which it is indicated that the solution (26) takes the shape of hyperbolic secant function with peak amplitude

$$
\mathcal{H}=2\left|\beta_{1}^{*}\right| \tilde{b}_{1} \mathrm{e}^{-\xi_{1}}
$$

and velocity

$$
\mathcal{V}=1024 \tilde{a}_{1}^{7}-7168 \tilde{a}_{1}^{5} \tilde{b}_{1}^{2}+7168 \tilde{a}_{1}^{3} \tilde{b}_{1}^{4}-1024 \tilde{a}_{1} \tilde{b}_{1}^{6}
$$

To show the localized structures and dynamic behaviors of one-soliton solution (26), we select the involved parameters as $\tilde{a}_{1}=0.3, \tilde{b}_{1}=0.25, \alpha_{1}=\beta_{1}=1, \xi_{1}=0$. The plots are depicted in Figs. 1-3. 


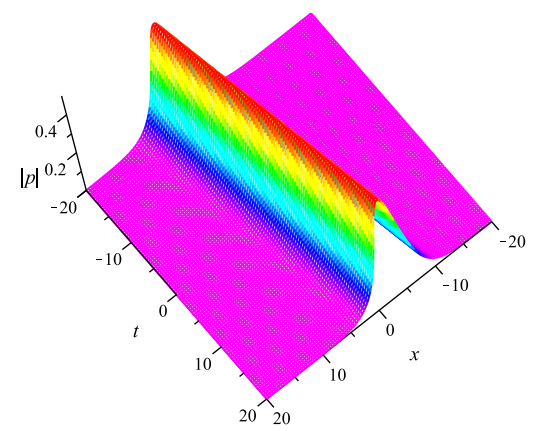

(a)

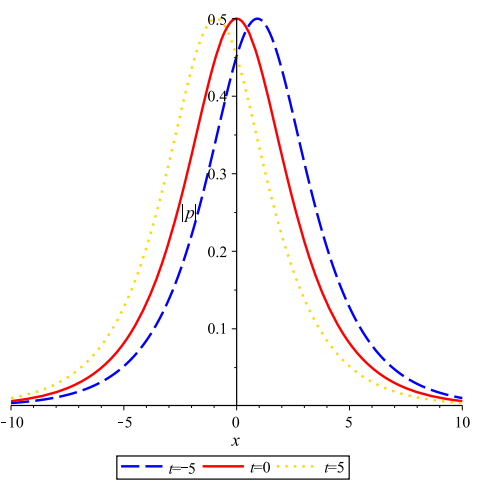

(b)

Figure 1 One-soliton solution (26) with $\tilde{a}_{1}=0.3, \tilde{b}_{1}=0.25, \alpha_{1}=\beta_{1}=1, \xi_{1}=0$. (a) Perspective view of modulus of $p$; (b) The soliton along the $x$-axis with different time in Fig. 1 (a)

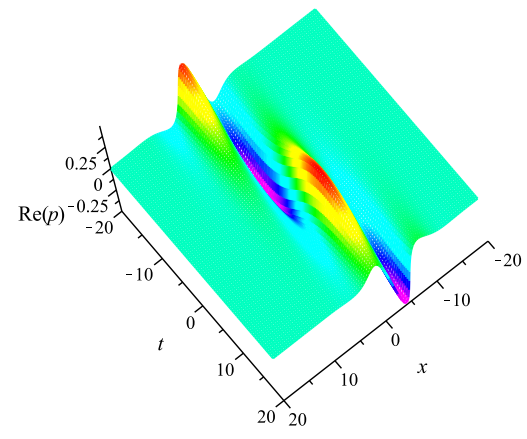

(a)

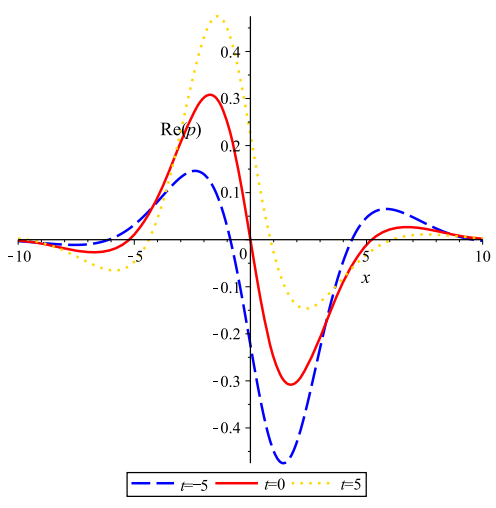

(b)

Figure 2 One-soliton solution (26) with $\tilde{a}_{1}=0.3, \tilde{b}_{1}=0.25, \alpha_{1}=\beta_{1}=1, \xi_{1}=0$. (a) Perspective view of the real part of $p$; (b) The soliton along the $x$-axis with different time in Fig. 2(a)

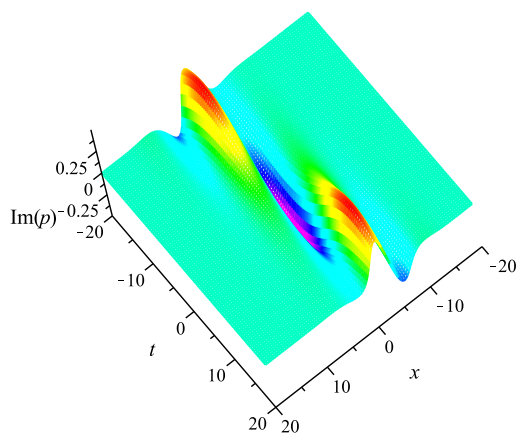

(a)

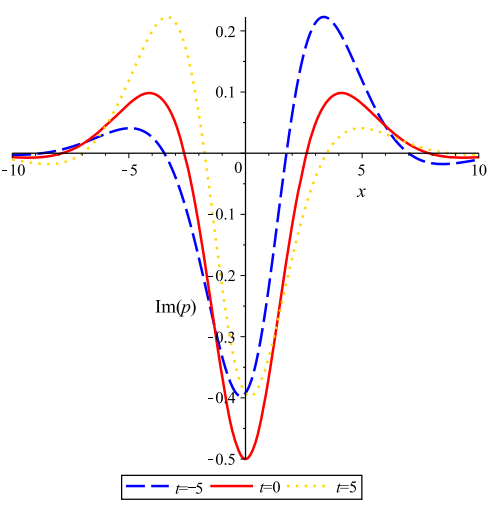

(b)

Figure 3 One-soliton solution (26) with $\tilde{a}_{1}=0.3, \tilde{b}_{1}=0.25, \alpha_{1}=\beta_{1}=1, \xi_{1}=0$. (a) Perspective view of the imaginary part of $p$; (b) The soliton along the $x$-axis with different time in Fig. 3(a) 
Then, for the case of $N=2$, the bright two-soliton solution for Equation (2) is generated as

$$
\begin{aligned}
p(x, t)= & \frac{2}{M_{12} M_{21}-M_{11} M_{22}}\left(\alpha_{1} \beta_{1}^{*} \mathrm{e}^{\theta_{1}^{*}-\theta_{1}} M_{22}-\alpha_{1} \beta_{2}^{*} \mathrm{e}^{\theta_{2}^{*}-\theta_{1}} M_{12}\right. \\
& \left.-\alpha_{2} \beta_{1}^{*} \mathrm{e}^{\theta_{1}^{*}-\theta_{2}} M_{21}+\alpha_{2} \beta_{2}^{*} \mathrm{e}^{\theta_{2}^{*}-\theta_{2}} M_{11}\right)
\end{aligned}
$$

where

$$
\begin{array}{ll}
M_{11}=\frac{\left|\alpha_{1}\right|^{2} \mathrm{e}^{-\theta_{1}^{*}-\theta_{1}}+\left|\beta_{1}\right|^{2} \mathrm{e}^{\theta_{1}^{*}+\theta_{1}}}{\varsigma_{1}-\varsigma_{1}^{*}}, & M_{12}=\frac{\alpha_{1}^{*} \alpha_{2} \mathrm{e}^{-\theta_{1}^{*}-\theta_{2}}+\beta_{1}^{*} \beta_{2} \mathrm{e}^{\theta_{1}^{*}+\theta_{2}}}{\varsigma_{2}-\varsigma_{1}^{*}}, \\
M_{21}=\frac{\alpha_{2}^{*} \alpha_{1} \mathrm{e}^{-\theta_{2}^{*}-\theta_{1}}+\beta_{2}^{*} \beta_{1} \mathrm{e}^{\theta_{2}^{*}+\theta_{1}}}{\varsigma_{1}-\varsigma_{2}^{*}}, & M_{22}=\frac{\left|\alpha_{2}\right|^{2} \mathrm{e}^{-\theta_{2}^{*}-\theta_{2}}+\left|\beta_{2}\right|^{2} \mathrm{e}_{2}^{\theta_{2}^{*}+\theta_{2}}}{\varsigma_{2}-\varsigma_{2}^{*}},
\end{array}
$$

and $\theta_{1}=i \varsigma_{1} x-128 i \varsigma_{1}^{8} t, \theta_{2}=i \varsigma_{2} x-128 i \varsigma_{2}^{8} t, \varsigma_{1}=\tilde{a}_{1}+i \tilde{b}_{1}, \varsigma_{2}=\tilde{a}_{2}+i \tilde{b}_{2}$.

After assuming that $\alpha_{1}=\alpha_{2}=1$ and $\beta_{1}=\beta_{2}$ as well as $\left|\beta_{1}\right|^{2}=\mathrm{e}^{2 \xi_{1}}$, the bright two-soliton solution (27) becomes

$$
\begin{aligned}
p(x, t)= & \frac{2}{M_{12} M_{21}-M_{11} M_{22}} \\
& \times\left(\beta_{1}^{*} \mathrm{e}^{\theta_{1}^{*}-\theta_{1}} M_{22}-\beta_{2}^{*} \mathrm{e}^{\theta_{2}^{*}-\theta_{1}} M_{12}-\beta_{1}^{*} \mathrm{e}^{\theta_{1}^{*}-\theta_{2}} M_{21}+\beta_{2}^{*} \mathrm{e}^{\theta_{2}^{*}-\theta_{2}} M_{11}\right),
\end{aligned}
$$

where

$$
\begin{aligned}
& M_{11}=-\frac{i}{\tilde{b}_{1}} \mathrm{e}^{\xi_{1}} \cosh \left(\theta_{1}^{*}+\theta_{1}+\xi_{1}\right), \\
& M_{12}=\frac{2 \mathrm{e}^{\xi_{1}}}{\left(\tilde{a}_{2}-\tilde{a}_{1}\right)+i\left(\tilde{b}_{1}+\tilde{b}_{2}\right)} \cosh \left(\theta_{1}^{*}+\theta_{2}+\xi_{1}\right), \\
& M_{21}=\frac{2 \mathrm{e}^{\xi_{1}}}{\left(\tilde{a}_{1}-\tilde{a}_{2}\right)+i\left(\tilde{b}_{1}+\tilde{b}_{2}\right)} \cosh \left(\theta_{2}^{*}+\theta_{1}+\xi_{1}\right), \\
& M_{22}=-\frac{i}{\tilde{b}_{2}} \mathrm{e}^{\xi_{1}} \cosh \left(\theta_{2}^{*}+\theta_{2}+\xi_{1}\right) .
\end{aligned}
$$

The localized structure and dynamic behaviors of two-soliton solution (28) are depicted in Fig. 4 via a selection of the parameters as follows: $\tilde{a}_{1}=0.3, \tilde{b}_{1}=\tilde{b}_{2}=0.2, \alpha_{1}=\alpha_{2}=\beta_{1}=$ $\beta_{2}=1, \tilde{a}_{2}=\xi_{1}=\xi_{2}=0$.

\section{Conclusion}

In this investigation, the aim was to explore multi-soliton solutions for an eighth-order nonlinear Schrödinger equation arising in an optical fiber. The method we resort to was the Riemann-Hilbert approach which is based on a matrix Riemann-Hilbert problem. Therefore, we first described a related Riemann-Hilbert problem via analyzing the spectral problem. After solving the resulting Riemann-Hilbert problem without reflection, we finally derived the expression of general $N$-soliton solution explicitly. We remark that this work mainly emphasizes the effectiveness of the Riemann-Hilbert method in dealing with higher-order nonlinear differential equation. Specifically, an eighth-order nonlinear Schrödinger equation is considered, which can also be generated from the AKNS hierarchy [29]. 


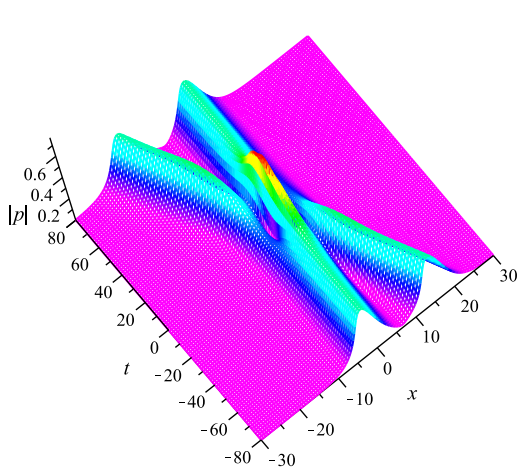

(a)

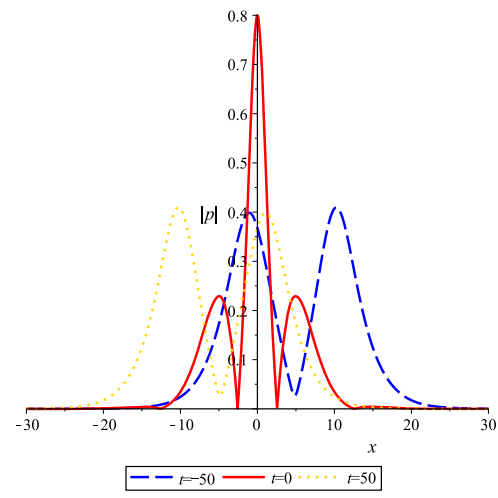

(b)

Figure 4 Two-soliton solution (28) with $\tilde{a}_{1}=0.3, \tilde{b}_{1}=\tilde{b}_{2}=0.2, \alpha_{1}=\alpha_{2}=\beta_{1}=\beta_{2}=1, \tilde{a}_{2}=\xi_{1}=\xi_{2}=0$. (a) Perspective view of modulus of $p$; (b) The soliton along the $x$-axis with different time in Fig. 4(a)

\section{Funding}

This work was supported by the National Natural Science Foundation of China (Grant Nos. 61072147 and 11271008).

\section{Competing interests}

The authors declare that they have no competing interests.

\section{Authors' contributions}

All authors contributed equally and significantly in writing this article. All authors read and approved the final manuscript.

\section{Author details}

${ }^{1}$ Department of Mathematics, Shanghai University, Shanghai, China. ${ }^{2}$ College of Mathematics, Inner Mongolia University for Nationalities, Tongliao, China. ${ }^{3}$ Department of Mathematics and Statistics, University of South Florida, Tampa, USA. ${ }^{4}$ Department of Mathematics, Zhejiang Normal University, Jinhua, China.

\section{Publisher's Note}

Springer Nature remains neutral with regard to jurisdictional claims in published maps and institutional affiliations.

Received: 16 October 2018 Accepted: 25 April 2019 Published online: 15 May 2019

\section{References}

1. Ankiewicz, A., Kedziora, D.J., Chowdury, A., Bandelow, U., Akhmediev, N.: Infinite hierarchy of nonlinear Schrödinger equations and their solutions. Phys. Rev. E 93, 012206 (2016)

2. Hirota, R.: Exact envelope-soliton solutions of a nonlinear wave equation. J. Math. Phys. 14, 805-809 (1973)

3. Ankiewicz, A., Soto-Crespo, J.M., Akhmediev, N.: Rogue waves and rational solutions of the Hirota equation. Phys. Rev. E 81, 046602 (2010)

4. Chen, S., Baronio, F., Soto-Crespo, J.M., Grelu, P., Mihalache, D.: Versatile rogue waves in scalar, vector, and multidimensional nonlinear systems. J. Phys. A, Math. Theor. 50, 463001 (2017)

5. Mihalache, D.: Multidimensional localized structures in optical and matter-wave media: a topical survey of recent literature. Rom. Rep. Phys. 69, 403 (2017)

6. Porsezian, K., Daniel, M., Lakshmanan, M.: On the integrability aspects of the one-dimensional classical continuum isotropic biquadratic Heisenberg spin chain. J. Math. Phys. 33, 1807-1816 (1992)

7. Yang, B., Zhang, W.G., Zhang, H.Q., Pei, S.B.: Generalized Darboux transformation and rogue wave solutions for the higher-order dispersive nonlinear Schrödinger equation. Phys. Scr. 88, 065004 (2013)

8. Chai, J., Tian, B., Zhen, H.L., Sun, W.R.: Conservation laws, bilinear forms and solitons for a fifth-order nonlinear Schrödinger equation for the attosecond pulses in an optical fiber. Ann. Phys. 359, 371-384 (2015)

9. Hu, W.Q., Gao, Y.T., Zhao, C., Feng, Y.J., Su, C.Q.: Oscillations in the interactions among multiple solitons in an optical fibre. Z. Naturforsch. 71, 1079-1091 (2016)

10. Hu, W.Q., Gao, Y.T., Zhao, C., Lan, Z.Z.: Breathers and rogue waves for an eighth-order nonlinear Schrödinger equation in an optical fiber. Mod. Phys. Lett. B 31, 1750035 (2017)

11. Inc, M., Aliyu, A.I., Yusuf, A., Baleanu, D.: Combined optical solitary waves and conservation laws for nonlinear Chen-Lee-Liu equation in optical fibers. Optik 158, 297-304 (2018)

12. Inc, M., Hashemi, M.S., Aliyu, A.l.: Exact solutions and conservation laws of the Bogoyavlenskii equation. Acta Phys. Pol. A 133, 1133-1137 (2018)

13. Inc, M., Aliyu, A.I., Yusuf, A., Baleanu, D.: Novel optical solitary waves and modulation instability analysis for the coupled nonlinear Schrödinger equation in monomode step-index optical fibers. Superlattices Microstruct. 113, 745-753 (2018)

14. Aliyu, A.I., Inc, M., Yusuf, A., Baleanu, D.: Symmetry analysis, explicit solutions, and conservation laws of a sixth-order nonlinear Ramani equation. Symmetry 10, 341 (2018) 
15. Aliyu, A.I., Inc, M., Yusuf, A., Baleanu, D.: Optical solitons and stability analysis in ring-cavity fiber system with carbon nanotube as saturable absorber. Commun. Theor. Phys. 70, 511-514 (2018)

16. Matveev, V.B., Smirnov, A.O.: AKNS and NLS hierarchies, MRW solutions, $P_{n}$ breathers, and beyond. J. Math. Phys. 59, 091419 (2018)

17. Mihalache, D., Panoiu, N.C., Moldoveanu, F., Baboiu, D.M.: The Riemann problem method for solving a perturbed nonlinear Schrödinger equation describing pulse propagation in optic fibres. J. Phys. A, Math. Gen. 27, 6177-6189 (1994)

18. Zhang, Y.S., Cheng, Y., He, J.S.: Riemann-Hilbert method and N-soliton for two-component Gerdjikov-Ivanov equation. J. Nonlinear Math. Phys. 24, 210-223 (2017)

19. de Monvel, A.B., Shepelsky, D.: A Riemann-Hilbert approach for the Degasperis-Procesi equation. Nonlinearity 26, $2081-2107(2013)$

20. de Monvel, A.B., Shepelsky, D.: The Ostrovsky-Vakhnenko equation by a Riemann-Hilbert approach. J. Phys. A, Math. Theor. 48, 035204 (2015)

21. Zhang, N., Hu, B.B., Xia, T.C.: A Riemann-Hilbert approach to complex Sharma-Tasso-Olver equation on half line. Commun. Theor. Phys. 68, 580-594 (2017)

22. Ma, W.X., Dong, H.H.: Modeling Riemann-Hilbert problems to get soliton solutions. Math. Model. Appl. 6, 16-25 (2017)

23. Hu, B.B., Xia, T.C., Zhang, N., Wang, J.B.: Initial-boundary value problems for the coupled higher-order nonlinear Schrödinger equations on the half-line. Int. J. Nonlinear Sci. Numer. Simul. 19, 83-92 (2018)

24. Hu, B.B., Xia, T.C., Ma, W.X.: Riemann-Hilbert approach for an initial-boundary value problem of the two-component modified Korteweg-de Vries equation on the half-line. Appl. Math. Comput. 332, 148-159 (2018)

25. Hu, B.B., Xia, T.C., Ma, W.X.: The Riemann-Hilbert approach to initial-boundary value problems for integrable coherently coupled nonlinear Schrödinger systems on the half-line. East Asian J. Appl. Math. 8, 531-548 (2018)

26. Ma, W.X.: Riemann-Hillbert problems and N-soliton solutions for a coupled mKdV system. J. Geom. Phys. 132, 45-54 (2018)

27. Ma, W.X: Riemann-Hilbert problems of a six-component fourth-order AKNS system and its soliton solutions. Comput. Appl. Math. 37, 6359-6375 (2018)

28. Guo, B.L., Liu, N., Wang, Y.F: A Riemann-Hilbert approach for a new type coupled nonlinear Schrödinger equations. J. Math. Anal. Appl. 459, 145-158 (2018)

29. Ma, W.X.: Application of the Riemann-Hilbert approach to the multicomponent AKNS integrable hierarchies. Nonlinear Anal., Real World Appl. 47, 1-17 (2019)

30. Yang, J.K.: Nonlinear Waves in Integrable and Nonintegrable Systems. SIAM, Philadelphia (2010)

\section{Submit your manuscript to a SpringerOpen ${ }^{\circ}$ journal and benefit from:}

- Convenient online submission

- Rigorous peer review

- Open access: articles freely available online

- High visibility within the field

- Retaining the copyright to your article

Submit your next manuscript at $\gg$ springeropen.com 